\title{
DYNAMICS OF CHEMICAL REACTIONS OF ASTROPHYSICAL INTEREST
}

\author{
M. ALAGIA, N. BALUCANI AND L. CARTECHINI
}

\author{
AND \\ P. CASAVECCHIA AND G.G. VOLPI \\ Dipartimento di Chimica, Università di Perugia \\ 06100 Perugia, Italy
}

\begin{abstract}
The dynamics of the astrophysically relevant reactions $\mathrm{OH}+$ $\mathrm{H}_{2}, \mathrm{OH}+\mathrm{CO}, \mathrm{N}\left({ }^{2} \mathrm{D}\right)+\mathrm{C}_{2} \mathrm{H}_{2}, \mathrm{~N}\left({ }^{2} \mathrm{D}\right)+\mathrm{H}_{2}$ and $\mathrm{O}\left({ }^{1} \mathrm{D}\right)+\mathrm{H}_{2}$ are studied using the crossed beam scattering technique. Comparisons of the experimental results with those of dynamics calculations on $a b$ initio and semi-empirical potential energy surfaces are discussed.
\end{abstract}

\section{Introduction}

During the past ten years it has been recognized that neutral-neutral reactions are about as important as ion-molecule reactions in interstellar and planetary chemistry, and this has prompted a great deal of experimental and theoretical studies on the neutral chemistry of the interstellar medium (ISM) and planetary atmospheres (Millar \& Williams 1988, Herbst 1995, Liao \& Herbst 1995, Flower 1995). A large variety of atoms, small radicals and molecules have been detected in these environments, whose densities and temperatures range from very low $\left(\mathrm{n}_{\mathrm{H}_{2}} \simeq 10^{3} / \mathrm{cm}^{3}, T \simeq 10 \mathrm{~K}\right)$ to quite large values $\left(\mathrm{n}_{\mathrm{H}_{2}} \simeq 10^{10} / \mathrm{cm}^{3}, T \geq 1000 \mathrm{~K}\right)$. Of course, mainly chemical reactions which have a negligible energy barrier, such as many radicalmolecule and radical-radical reactions, will play a significant role in low density and low temperature regions. However, reactions with some energy barrier become important too in dense and warm regions and interstellar shock-heated gas. Detailed work on the kinetics (rate coefficients as functions of temperature) of astrophysically relevant neutral-neutral reactions has recently been undertaken down to very low temperatures (Smith 1995, 
1996). Nevertheless, for reliable astrophysical chemical modelling there is a need to know not only the reaction rates, but also the primary reaction products, their internal energy and the dynamics of their formation.

Tremendous progress has occurred over the past several years in the study of the dynamics of atom and small radical reactions (Liu \& Wagner 1995) following impressive advancements in molecular beam and laser spectroscopic techniques, as well as quantum reactive scattering methods and electronic structure calculations. The effort to understand the behaviour of atom and small radical reactions is motivated by the great relevance they bear in many areas - from Earth's to planetary atmosphere chemistry, from combustion to interstellar cloud chemistry - in addition to their fundamental interest. Laboratory experiments suitable for investigating the dynamics of astrophysically important reactions have now become possible thanks to the development of laser photolysis (Patel-Misra \& Dagdigian 1992, Che \& Liu 1995), laser ablation (Kaiser et al. 1995) and electrical discharge (Alagia et al. 1995) techniques for generating radical beams.

In this contribution, we will outline some recent progress in the dynamical study of reactions of interest in astrophysics, that has been achieved by using the crossed molecular beam (CMB) scattering method with rotating mass spectrometric detection, which is one of the exciting experimental advances that has given rise to a new level of understanding of chemical reaction dynamics (Lee 1987a,b). We will consider here reactions of hydroxyl radicals and of electronically excited oxygen and nitrogen atoms.

$\mathrm{OH}$ radicals are very important in the interstellar and circumstellar environment; they mostly originate from $\mathrm{H}_{2} \mathrm{O}$ photodissociation. In diffuse clouds, $\mathrm{OH}$ abundances may locally be enhanced by shock waves through the reaction $\mathrm{O}\left({ }^{3} \mathrm{P}\right)+\mathrm{H}_{2} \rightarrow \mathrm{OH}+\mathrm{H}$. The newly formed $\mathrm{OH}$ is then converted to water through the reaction:

$$
\mathrm{OH}+\mathrm{H}_{2} \rightarrow \mathrm{H}_{2} \mathrm{O}+\mathrm{H} \quad \Delta \mathrm{H}_{0}^{\circ}=-61.9 \mathrm{~kJ} / \mathrm{mol}
$$

This reaction may also play some role as regeneration step of the $\mathrm{OH}$ interstellar maser in star formation regions (Andresen 1986) and could be of some importance as active medium of $\mathrm{H}_{2} \mathrm{O}$ masers. In general, abstraction reactions of atoms and radicals with $\mathrm{H}_{2}$ are important in the synthesis of interstellar molecules, since $\mathrm{H}_{2}$ is by far the most abundant molecule in ISM. Another important reaction of $\mathrm{OH}$ is that with $\mathrm{CO}$, the second most abundant interstellar molecule:

$$
\mathrm{OH}+\mathrm{CO} \rightarrow \mathrm{CO}_{2}+\mathrm{H} \quad \Delta \mathrm{H}_{0}^{\circ}=-102.5 \mathrm{~kJ} / \mathrm{mol}
$$

which may lead to significant concentrations of $\mathrm{CO}_{2}$ in dense interstellar clouds where $\mathrm{OH}$ and $\mathrm{CO}$ are relatively abundant (Prasad \& Huntress 1980). This reaction has also been considered as part of the chemistry of 
carbon-bearing molecules in oxygen-rich circumstellar envelopes (Nejad \& Millar 1988).

Oxygen and nitrogen atoms in the first electronically excited state, $\mathrm{O}\left({ }^{1} \mathrm{D}\right)$ and $\mathrm{N}\left({ }^{2} \mathrm{D}\right)$, are metastable species with lifetimes of the order of $150 \mathrm{~s}$ and $40 \mathrm{~h}$, respectively, and originate from photodissociation, electronimpact dissociation and dissociative recombination processes. The relevance of their chemistry to several planetary atmospheres was pointed out by Dalgarno (1988); moreover, reactions of $\mathrm{O}\left({ }^{1} \mathrm{D}\right)$ and $\mathrm{N}\left({ }^{2} \mathrm{D}\right)$ may be relevant in dense and hot interstellar regions, where excited species can be formed and react before they radiatively decay. We have recently investigated the reactions of $\mathrm{O}\left({ }^{1} \mathrm{D}\right)$ with $\mathrm{H}_{2}$ and of $\mathrm{N}\left({ }^{2} \mathrm{D}\right)$ with $\mathrm{H}_{2}$ and $\mathrm{C}_{2} \mathrm{H}_{2}$, the latter species also abundant in interstellar space. While complementary information on the dynamics of some $\mathrm{O}\left({ }^{1} \mathrm{D}\right)$ reactions comes from pump-probe time-resolved spectroscopic studies (Aker \& Sloan 1986, Cleveland et al. 1987), no such studies have been carried out to date on the dynamics of $\mathrm{N}\left({ }^{2} \mathrm{D}\right)$ and $\mathrm{OH}$ reactions.

Elementary reactions of $\mathrm{OH}$ radicals and $\mathrm{O}\left({ }^{1} \mathrm{D}\right)$ have attracted large theoretical interest over the years (Clary 1994, Peng et al. 1996). In particular, semi-empirical and $a b$ initio potential energy surfaces (PESs) have been developed and dynamical calculations, both classical and quantummechanical, have been carried out on them (see references in Alagia et al. 1995 and Casavecchia et al. 1995).

After a brief description of the experimental technique, we will present a survey of experimental results and emphasize the detailed comparison between them and the results of state-of-the-art quantum (approximate) as well as quasiclassical dynamical calculations on $a b$ initio and/or semiempirical PESs in an effort to assess the current status of theory versus experiment for simple radical-molecule reactions of astrophysical interest.

\section{Experimental method}

A cross sectional view from the top of our crossed molecular beam apparatus (Alagia et al. 1995) is shown in Fig. 1. Briefly, two supersonic beams of reagents, well collimated in angle and velocity, are crossed at $90^{\circ}$ in a large scattering chamber kept at $10^{-7}$ mbar, i.e., under single collision conditions. A quadrupole mass spectrometer, contained in a ultra-high-vacuum region $\left(10^{-11} \mathrm{mbar}\right)$, serves as detector of the reaction products. It can be rotated in the plane defined by the two beams around their intersection axis in order to measure the product angular distribution. The velocities of the products (i.e., their translational energy) can be derived from time-of-flight (TOF) measurements. By energy conservation also the product internal energy is 


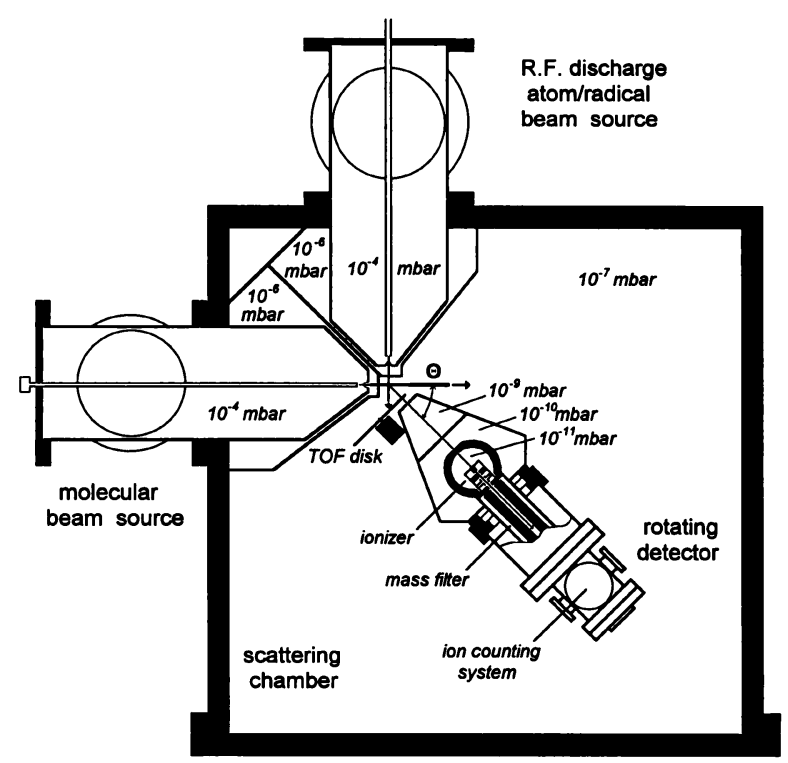

Figure 1. Top-view (cross sectional) of the crossed molecular beam apparatus, showing the scattering chamber, the differentially pumped beam sources and electron-impact quadrupole mass spectrometer detector, and the time-of-flight chopper.

derived. In these experiments, a selection of the velocities, as well as internal quantum states, of the reactants is achieved by supersonic expansion. The key to the success of these experiments is the generation of intense beams of the unstable species (atoms or radicals): we use a versatile beam source based on a high-power radio-frequency discharge through a quartz nozzle of dilute gas mixtures of suitable precursor molecules $\left(\mathrm{O}_{2}\right.$ and $\mathrm{N}_{2}$ for $\mathrm{O}$ and $\mathrm{N}$ atoms, and $\mathrm{H}_{2} \mathrm{O}$ for $\mathrm{OH}$ radicals) in rare gases (Casavecchia et al. 1995).

\section{Experimental results and comparison with theory}

\subsection{THE REACTION $\mathrm{OH}+\mathrm{H}_{2}$}

The reaction of the ubiquitous $\mathrm{OH}$ radical with $\mathrm{H}_{2}$ is of considerable relevance not only in astrochemistry but in combustion and atmospheric chemistry as well. Due to its simplicity (it involves three hydrogen atoms) it is amenable to detailed theoretical treatment and, indeed, it has taken a prototype role in the field of reaction dynamics (Clary 1994). While inves- 

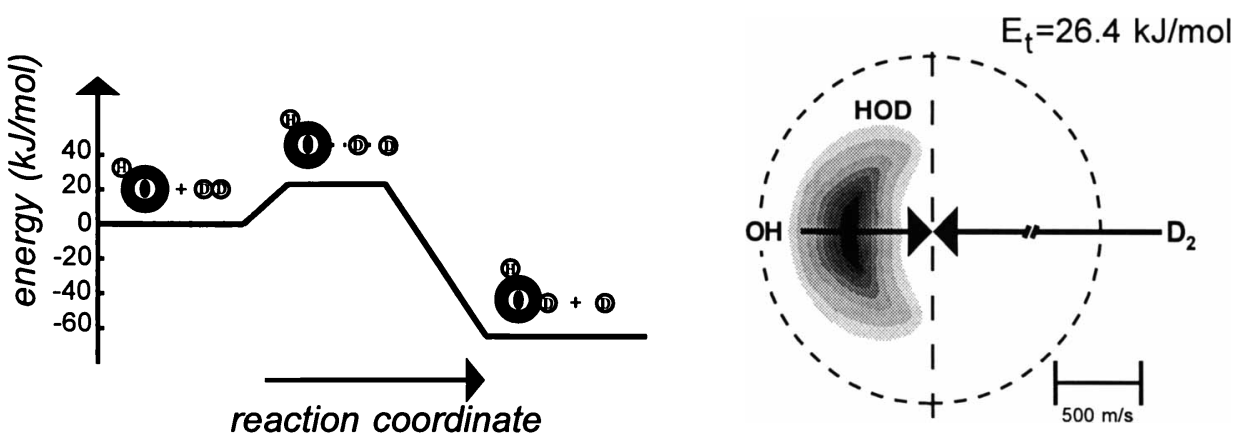

Figure 2. Left: Potential energy profile along the minimum energy path of the reaction $\mathrm{OH}+\mathrm{D}_{2} \rightarrow \mathrm{HOD}+\mathrm{D}$, as obtained from ab initio calculations. Right: HOD product flux map for the $\mathrm{OH}+\mathrm{D}_{2}$ reaction at $\mathrm{E}_{t}=26.4 \mathrm{~kJ} / \mathrm{mol}$. The polar map shows the probability of HOD appearing at specific angles and velocities in the center-of-mass system. The colors, going from light grey to black, indicate increasing intensities.

tigation of its kinetics dates back to the mid 1970s, only during the last few years it has become possible to tackle its dynamics experimentally (Alagia et al. 1996). From angular and velocity distribution measurements of the $\mathrm{HOD}$ product formed from the isotopic variant $\mathrm{OH}+\mathrm{D}_{2}$ reaction at a translational energy $\left(\mathrm{E}_{t}\right)$ of $26.4 \mathrm{~kJ} / \mathrm{mol}$, we have derived the product flux contour map in the center-of-mass reference frame (Fig. 2), which represents how the product is distributed in angle and velocity space after the reactive collision. This quantity, technically called the double differential cross section, can be compared with the prediction of a PES through scattering calculations based on quasiclassical trajectory (QCT) and quantum mechanical methods, the latter within the so-called rotating bond approximation (Clary 1991, 1992). We found that the HOD product is scattered in the backward direction with respect to the incoming $\mathrm{OH}$ (see Fig. 2); this indicates that the reaction is direct, i.e., it takes place on a very short time-scale (of the order of molecular vibrations), and that the most favorable orientation for reaction is collinear, or nearly collinear. From where the distribution peaks on the velocity scale we derive that $34 \%$ of the total available energy (relative translational energy + reaction exoergicity) is released as translational motion of products. Since for this collinearly dominated reaction one does not expect much product rotational excitation, a modest translational energy release implies, by energy conservation, that HOD is highly vibrationally excited, that is, infrared active. The product energy partitioning is very sensitive to the characteristics of the PES, especially in the critical region of the transition state. We have found (Alagia et al. 1996) that a very recent PES (Kliesch et al. 1996), obtained from large scale electronic structure calculations, represents a significant improvement with respect to the previous existing PES (Schatz \& Elgersma 1980): the 

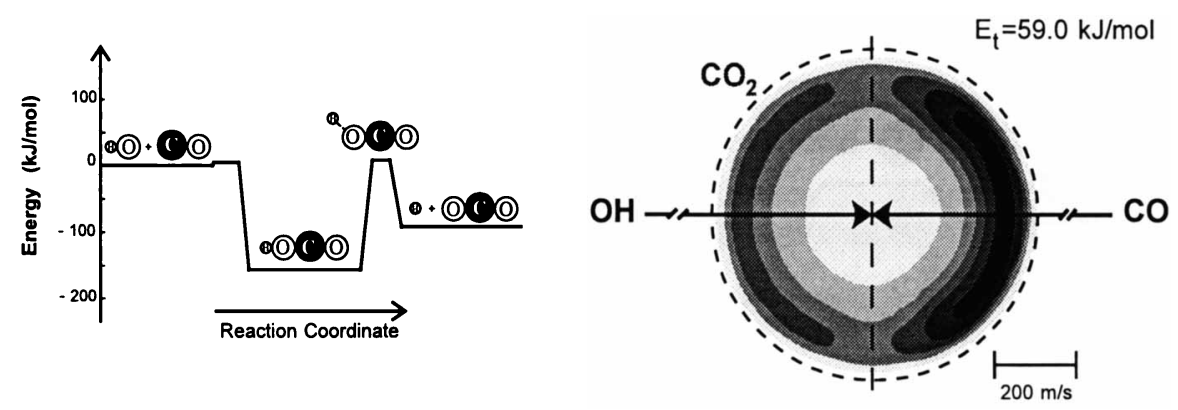

Figure 3. Left: Potential energy profile along the minimum energy path of the reaction $\mathrm{OH}+\mathrm{CO} \rightarrow \mathrm{CO}_{2}+\mathrm{H}$, as obtained from ab initio calculations. Right: $\mathrm{CO}_{2}$ product flux map for the $\mathrm{OH}+\mathrm{CO}$ reaction at $\mathrm{E}_{t}=59.0 \mathrm{~kJ} / \mathrm{mol}$.

new PES predicts about $44 \%$ of the available energy in product translation while the old one predicts $71 \%$.

Although the remaining disagreement points to some inadequacies of the PES, exact quantum scattering calculations are in order for an unambiguous test of the $a b$ initio surface.

\subsection{THE REACTION OH+CO}

The reaction of the $\mathrm{OH}$ radical with $\mathrm{CO}$ is again of great relevance also in combustion and atmospheric chemistry. From a fundamental point of view, it is a prototype of four-atom reactions with a deep potential well between reactants and products (see Fig. 3, where the reaction coordinate diagram is shown as indicated by ab initio calculations of the PES (Kudla et al. 1991)). This reaction is perhaps the most studied, from a kinetic standpoint, radical-molecule reaction (Fulle et al. 1996). Very recently, we have been able to investigate its dynamics in crossed beams at two different relative translational energies (Casavecchia et al. 1995, Alagia et al. 1995). From angular and velocity distribution measurements at $\mathrm{E}_{t}=59.0 \mathrm{~kJ} / \mathrm{mol}$, the product flux contour map shown in Fig. 3 has been derived. As can be seen, the dynamics of $\mathrm{CO}_{2}$ formation are very different from that of HOD from $\mathrm{OH}+\mathrm{D}_{2}$ (see Fig. 2). In fact, the formed $\mathrm{CO}_{2}$ is not confined in one of the two hemispheres, but is scattered both in the backward and forward direction, with a small preference for the forward one; another difference is that the flux distribution extends to the limits of energy conservation (the maximum velocity achievable by the product is represented by the dashed circle in Fig. 3). These findings witness the formation of a HOCO complex whose lifetime is of the order of its rotational period (about one picosecond) and a high product translational excitation. Moreover, since the product angular distribution is nearly isotropic, a high product rotational excitation 
is inferred. Indeed, it is found that about $65 \%$ of the available energy is released as recoil energy of the products; this indicates that strong repulsive forces are abruptly exerted as the HOCO intermediate has overpassed the potential barrier in the exit channel (see Fig. 3) and the potential energy is mainly converted into kinetic energy. These results are a very sensitive probe of the PES. Comparison of the experimental results with those of QCT (Kudla \& Schatz 1995) and approximate quantum scattering calculations (Clary \& Schatz 1993) on the ab initio PES show good agreement for the product translational energy distribution, but less good agreement for the product angular distribution (Casavecchia et al. 1995). It is concluded that improved calculations of the PES as well as exact quantum scattering calculations of the dynamics are necessary.

\subsection{THE REACTION N($\left.{ }^{2} \mathrm{D}\right)+\mathrm{C}_{2} \mathrm{H}_{2}$}

The recent development of an efficient beam source of nitrogen atoms has allowed us, for the first time, to investigate the dynamics of some $\mathrm{N}$-atom reactions of importance in astrochemistry. The first reaction we have looked at is $\mathrm{N}\left({ }^{4} \mathrm{~S},{ }^{2} \mathrm{D}\right)+\mathrm{C}_{2} \mathrm{H}_{2}$, by using beams containing about $72 \%$ of $\mathrm{N}\left({ }^{4} \mathrm{~S}\right)$, $21 \%$ of $\mathrm{N}\left({ }^{2} \mathrm{D}\right)$ and $7 \% \mathrm{~N}\left({ }^{2} \mathrm{P}\right)$. We found that the primary product is HCCN (cyanomethylene) coming from the reaction:

$$
\mathrm{N}\left({ }^{2} \mathrm{D}\right)+\mathrm{C}_{2} \mathrm{H}_{2} \rightarrow \mathrm{HCCN}+\mathrm{H} \quad \Delta \mathrm{H}_{0}^{\circ}=-230 \mathrm{~kJ} / \mathrm{mol}
$$

No evidence was found of the reaction pathway leading to $\mathrm{CCN}+\mathrm{H}_{2}$. Fig. 4 shows the angular distribution measured at the mass-to-charge $(\mathrm{m} / \mathrm{e})$ ratio of $39\left(\mathrm{HCCN}^{+}\right)$and $38\left(\mathrm{CCN}^{+}\right)$at $\mathrm{E}_{t}=39.0 \mathrm{~kJ} / \mathrm{mol}$. The two distributions are identical, a fact which indicates that $\mathrm{CCN}^{+}$is the daughter ion coming from fragmentation of the parent ion $\mathrm{HCCN}^{+}$inside the electron impact ionizer of the mass spectrometer detector. From angular and velocity distribution measurements of the HCCN product at two different translational energies $(13.0$ and $39.0 \mathrm{~kJ} / \mathrm{mol})$, it is concluded that the reaction proceeds through the formation of a long-lived cyanomethylene $\left(\mathrm{H}_{2} \mathrm{CCN}\right)$ intermediate. The fraction of total available energy released in translation is modest $(<30 \%)$, which indicates that the HCCN radical is internally very excited. Cyanomethyl has been observed in the interstellar environment (in Sgr B2 and TMC-1) (Cummings et al. 1986, Turner et al. 1990), while cyanomethylene has been proposed as an important intermediate produced through reaction (3) in the atmosphere of Titan that leads to the formation of nitriles (Yung 1987). It may also be important in interstellar clouds and circumstellar envelopes, especially in carbon-rich stars. In general, reactions of $\mathrm{N}$ atoms are relevant in the chemistry of dark interstellar clouds because of the relatively high abundance of these atoms. A call was made (Yung 1987) for determining the rate constant of reaction 

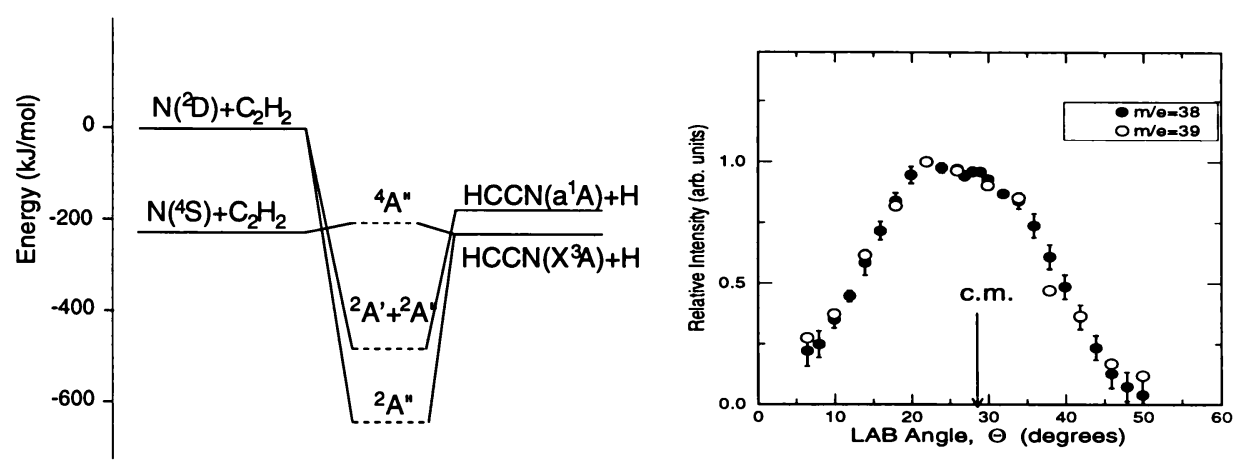

Figure 4. Correlation diagram for the $\mathrm{N}\left({ }^{4} \mathrm{~S},{ }^{2} \mathrm{D}\right)+\mathrm{C}_{2} \mathrm{H}_{2}$ reaction. Right: Laboratory angular distribution of the $\mathrm{HCCN}$ product (detected at $\mathrm{m} / \mathrm{e}=38$ and $\mathrm{m} / \mathrm{e}=39$ ) at $\mathrm{E}_{t}=29.0$ $\mathrm{kJ} / \mathrm{mol}$.

(3) to assess its relevance in the proposed Titan model (the estimated value of the model is $1 \cdot 10^{-11} \mathrm{~cm}^{3}$ molecule $\mathrm{c}^{-1} \mathrm{~s}^{-1}$ at $300 \mathrm{~K}$ ). From the HCCN intensity observed in the $\mathrm{CMB}$ experiments it is inferred that the reaction is quite fast $\left(k>1 \cdot 10^{-11} \mathrm{~cm}^{3}\right.$ molecule $\left.{ }^{-1} \mathrm{~s}^{-1}\right)$ at the temperature $(>1000 \mathrm{~K})$ corresponding to the relative translational energy of the experiment. Since reaction (3) is strongly exoergic and is expected to proceed with negligible energy barrier, it may be fast at lower temperatures too. These findings appear to support the chemistry proposed by Yung (1987) and establish that reaction (3) is an important process in the atmosphere of Titan.

\subsection{THE REACTIONS $\mathrm{N}\left({ }^{2} \mathrm{D}\right)+\mathrm{H}_{2}$ AND O $\left({ }^{1} \mathrm{D}\right)+\mathrm{H}_{2}$}

We have also investigated the reactions

$$
\begin{array}{ll}
\mathrm{N}\left({ }^{2} \mathrm{D}\right)+\mathrm{H}_{2} \rightarrow \mathrm{NH}+\mathrm{H} & \Delta \mathrm{H}_{0}^{\circ}=-138.9 \mathrm{~kJ} / \mathrm{mol} \\
\mathrm{O}\left({ }^{1} \mathrm{D}\right)+\mathrm{H}_{2} \rightarrow \mathrm{OH}+\mathrm{H} & \Delta \mathrm{H}_{0}^{\circ}=-182.0 \mathrm{~kJ} / \mathrm{mol}
\end{array}
$$

at low energies $(8 \div 22 \mathrm{~kJ} / \mathrm{mol})$ and found that both proceed mainly through insertion of the excited atom into the $\mathrm{H}_{2}$ bond forming short-lived intermediates $\left(\mathrm{NH}_{2}\right.$ and $\mathrm{H}_{2} \mathrm{O}$, respectively), which live a few bending vibrations before decomposing into $\mathrm{NH}+\mathrm{H}$ and $\mathrm{OH}+\mathrm{H}$, respectively, without time for energy randomization. The modest fraction (about $30 \%$ ) of available energy found in product translational energy indicates a very high internal excitation of the radical product.

While for $\mathrm{O}\left({ }^{1} \mathrm{D}\right)+\mathrm{H}_{2}$ comparison of experimental results with theoretical predictions (QCT calculations on a semi-empirical PES) is not satisfactory and calls for more accurate calculations of the PES (Alagia et al. 1997), for $\mathrm{N}\left({ }^{2} \mathrm{D}\right)+\mathrm{H}_{2}$ comparison between theory and experiment may 
become possible in the near future following recent calculations of ab initio PESs for $\mathrm{NH}_{2}$ (Vetter et al. 1996, van Hemert et al. 1996).

These examples show that it is now possible to investigate the dynamics of elementary reactions of importance in astrophysics both experimentally and theoretically. A synergistic collaboration between experiment and theory is most fruitful.

\section{References}

Alagia, M., Balucani, N., Casavecchia, P., Stranges, D., Volpi, G.G. 1995, J. Chem. Soc. Faraday Trans. 91, 575 .

Alagia, M., Balucani, N., Casavecchia, P., Stranges, D., Volpi, G.G., Clary, D.C., Kliesch, A., Werner, H.-J. 1996, Chem. Phys. 207, 389.

Alagia, M., Balucani, N., Cartechini, L., Casavecchia, P., van Kleef, E.H., Volpi, G.G., Kuntz, P.J., Sloan, J.J. 1997, J. Chem. Phys., in preparation.

Aker, P.M. Sloan, J.J. 1986, J. Chem. Phys. 85, 1412.

Andresen, P. 1986, Astron. Astrophys. 154, 42; Comments At. Mol. Phys. 18, 1.

Dalgarno, A. 1988, in Rate Coefficients in Astrochemistry, Millar, T.J. and Williams, D.A. (eds.), (Kluwer, Dordrecht), p. 321.

Casavecchia P., Balucani, N., Volpi, G.G. 1995, in Advanced Series in Chemical Physics - Vol. 6, The Chemical Dynamics and Kinetics of Small Radicals (World Scientific, Singapore), ch. 9.

Che, D.-C., Liu, K. 1995, Chem. Phys. Lett. 243, 290.

Clary, D.C. 1991, J. Chem. Phys. 95, 7298.

Clary, D.C. 1992, J. Chem. Phys. 96, 3656.

Clary, D.C., Schatz, G.C. 1993, J. Chem. Phys. 99, 4578.

Clary, D.C. 1994, J. Phys. Chem. 98, 10678, and references therein.

Cleveland, C.B., Jurish, G.M., Trolier, M., Wiesenfeld, J.R. 1987, J. Chem. Phys. 86, 3253.

Cummings, S.E., Linke, R.A., Thaddeus, P. 1986, Astrophys. J. Suppl. 60, 819.

Flower, D.R. 1995, Int. Rev. Phys. Chem. 14, 421.

Fulle, D., Hamann, H.F., Hippler, H., Troe, J. 1996, J. Chem. Phys. 105, 983.

Herbst, E. 1995, Ann. Rev. Phys. Chem. 46, 27.

Kaiser, R.I., Lee, Y.T., Suits, A.G. 1995, J. Chem. Phys. 103, 10395.

Kliesch, A., Clary, D.C., Werner, H.-J. 1996, to be published.

Kudla, K., Schatz, G.C. 1995, in Advanced Series in Chemical Physics - Vol. 6, The Chemical Dynamics and Kinetics of Small Radicals, (World Scientific, Singapore), ch. 10.

Kudla, K., Schatz, G.C., Wagner, A.F. 1991, J. Chem. Phys. 95, 1635.

Lee, Y.T. 1987a, in Atomic and Molecular Beam Methods, ed. G. Scoles (Oxford, New York), vol.1.

Lee, Y.T. 1987b, Science 236, 793.

Liao, Q., Herbst, E. 1995, Astrophys. J. 444, 694.

Liu, K., Wagner, A. (eds.) 1995, Advanced Series in Physical Chemistry - Vol. 6, The Chemical Dynamics and Kinetics of Small Radicals (World Scientific, Singapore).

Millar, T.J., Williams, D.A. (eds.) 1988, Rate Coefficients in Astrochemistry (Kluwer, Dordrecht).

Nejad, L.A.M., Millar, T.J. 1988, Mon. Not. R. Astr. Soc. 230, 79.

Patel-Misra, D., Dagdigian, P.J. 1992, J. Chem. Phys. 96, 3232.

Peng, T., Zhang, D.H., Zhang, J.Z.H., Schinke, R. 1996, Chem. Phys. Lett. 248, 37, and references therein.

Prasad, S.S., Huntress, W.T. 1980, Astrophys. J. Suppl. 43, 1. 
Schatz, G.C., Elgersma, H. 1980, Chem. Phys. Lett. 73, 21.

Smith, I.W.M. 1995, Int. J. Mass Spectrom. and Ion Proc. 149/150, 231, and references therein; see also this volume.

Turner, B.E., Friberg, P., Irvine, W.M., Saito, S., Yamamoto, S. 1990, Astrophys. J. 355, 546.

van Hemert, M.C., Vetter, R., Kroes, G.J., van Dishoeck, E.F. 1996 in: IAU Symposium 178, Molecules in Astrophysics: Probes and Processes Book of Abstracts, eds. D.J. Jansen, M.R. Hogerheijde and E.F. van Dishoeck (Leiden Observatory), p.190.

Vetter, R., Zülicke, L., Koch, A., van Dishoeck, E.F., Peyerimhoff, S.D. 1996, J. Chem. Phys. 104, 5558.

Yung, Y. 1987, Icarus 72, 4668.

\section{Discussion}

Herbst: What is the minimum collision energy you can study?

Casavecchia: In our crossed molecular beam scattering experiments it is posssible to vary the collision energy by varying the beam velocities. This is achieved by changing the seeding gas and the nozzle temperature. The minimum collision energy that can be reached depends on the system under study. For $\mathrm{O}\left({ }^{1} \mathrm{D}\right)+\mathrm{H}_{2}$ and $\mathrm{N}\left({ }^{2} \mathrm{D}\right)+\mathrm{H}_{2}$ it is possible to go down to $1 \mathrm{Kcal} / \mathrm{mol}$. By varying the crossing angle of the two beams, which in principle is possible, the collision energy can be further decreased by a factor of several. 